\title{
A STUDY TO CORRELATE VSS AND VHQ IN PATIENTS WITH VERTIGO DUE TO PERIPHERAL VESTIBULAR DISORDERS
}

\author{
Pragna Landge ${ }^{* 1}$, Neha Patel ${ }^{2}$. \\ ${ }^{*}$ Assistant Professor, BITS Institute of Physiotherapy Varnama Vadodara, Gujarat, India. \\ ${ }^{2}$ Neurophysiotherapist, Gurugram Haryana India.
}

\section{ABSTRACT}

\begin{abstract}
There are ample of studies on use of VSS in order to find out the severity of vertigo symptoms, also studies portray the usefulness of $\mathrm{VHQ}$ to study the impact of vertigo on lifestyle; however, there is dearth of information on whether there is any correlation between the vertigo symptoms and affection on quality of life (QOL). Hence, the need arises to find out the correlation between VSS and VHQ for the better, documentation of symptoms and affection on QOL, as it will benefit treatment efficacy. METHODS: Sample size was 40. All the patients were given two questionnaires VSS and VHQ, which are self-administered. RESULTS: Correlation was found between total VSS and its dimensions with VHQ using Karl Pearson correlation coefficient method. Thus, we concluded that increase or decrease in the scores of VSS has an effect on the scores of VHQ.
\end{abstract}

KEY WORDS: Vertigo, Vertigo symptom scale (VSS), Vertigo handicap questionnaire (VHQ), Peripheral Vestibular disorder.

Address for correspondence: Dr. Pragna Landge MPT (ADULT NEUROLOGY AND PSYCHOSOMATIC DISORDERS), Assistant Professor BITS Institute of Physiotherapy Varnama Vadodara, Gujarat, India.

E-Mail: pragna3609@gmail.com

Access this Article online

Quick Response code

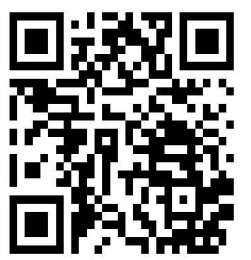

DOI: $10.16965 /$ ijpr.2019.128

Journal Information

International Journal of Physiotherapy and Research

ICV for 2016 ISSN (E) 2321-1822 | ISSN (P) 2321-8975

86.93 https://www.ijmhr.org/ijpr.html

DOI-Prefix: https://dx.doi.org/10.16965/ijpr

(cc) EY-No-Sh

Article Information

Received: 15 Mar 2019

Peer Review: 15 Mar 2019

Revised: None
Accepted: 22 Apr 2019

Published (O): 11 Jun 2019

Published (P): 11 Jun 2019

\section{INTRODUCTION}

Attacks of vertigo can be distressing due to autonomic and vegetative symptoms and are associated with anxiety, panic, decreased independence in activities in daily living including self-care skills and instrumental activities, decreased job performance, decreased participation in family responsibilities, and reluctance to participate in social activities. It will lead to the secondary problems such as decreased activity level, fatigue, headache, fear of falling. Thus, even though most vestibular disorders are benign and not life threatening, but it has serious consequences in the form of psychological sequel leading to negative and maladaptive coping mechanism such as avoidance of stimulation or reduces compliance to vestibular therapy and thus delays the recovery. Thus, it is necessary to investigate the vestibular symptom and psychological impact for the patient of vertigo [1].

A variety of questionnaires has been used in clinical practice to assess the symptoms and handicap along with anxiety. One of the benefits of using questionnaire is that it allows the clinician to get an idea regarding the patients thought on their complaints. Thus, prior to consultation the clinician can highlight relevant issues and can focus on patients thought related to their complaints. Skillfully selected questionnaires add to the completeness of case history and results may signal the 
need for further investigation or referrals.

One of such questionnaire is Vertigo symptom scale (VSS); it is a disease specific instrument. VSS can discriminate between the healthy individuals and patients with vertigo [2]. VSS has been researched extensively and concurrent validity and good reliability has been established. Predictive validity has been reported only for the VSS questionnaire. VSS is a self-assessment scale, which targets vestibular symptoms and has been translated in various languages without losing validity. The VSS has been translated into seven other languages, apart from English; Dutch, French, German, Spanish, Swedish, Turkish and Malay $[1,3,4]$.

The Vertigo symptom scale (VSS) by Yardley et $a l$. is one of the disease- specific subjective questionnaires to quantify balance disorder, somatic anxiety, and autonomic severity symptoms. ${ }^{15}$ As mentioned previously, the VSS is a well-known tool for the assessment of vestibular disorders and the associated symptoms of autonomic arousal and somatosensation. VSS also focuses on all the primary and secondary symptoms of vestibular disorder, including anxiety and autonomic symptoms $[4,5]$.

One of other questionnaire to assess the quality of life (or handicap) in patients with vertigo is (VHQ). VHQ was developed from the accounts of most common psychological consequences of vertigo. This scale has shown reliability and validity. VHQ was found to be one of the most relevant and promising questionnaires for assessing impact of vertigo on QOL $[6,7]$.

It has been proved in research that Vertigo symptom scale has a predictive validity and it is a reliable and valid tool. Vertigo affects quality of life, there are various studies showing the impact of vertigo on QOL example a study was carried out to evaluate the burden of dizziness and vertigo in community, it was found that particularly vestibular vertigo patients have lowered quality of life [8]. Therefore the aim of the study is to find if there is any correlation between severity of vertigo (VSS) and QOL (VHQ).

\section{METHODS}

Inclusion Criteria: The patient with peripheral vestibular disorder diagnosed by a specialist in
ENT/ Neurophysician. The patients with at least three spontaneous attacks of vertigo during the last year and /or were constantly unsteady during the last 6 months for at least $75 \%$ of the time when awake. The patients with a sufficient knowledge of the Kannada or English language and the physical and mental ability to understand and answer the questions.

Exclusion criteria: Patient should not have symptoms of vertigo secondary to central nervous system pathology. The patient should not have been in psychiatric care or on psychopharmacy.

Procedure: Ethical Clearance has been obtained from S.D.M. college of Medical Science And Hospital. The patients (outpatient / inpatient) with complaints of vertigo secondary to peripheral vestibular disorder referred by the Neurophysicians or specialists in ENT were included in the study. The patients who met the inclusion criteria were included in the study. A Pilot study was conducted for the reliability and validity of the translated version of VSS and VHQ (in Kannada language) questionnaires $(r=0.791)$. The study was briefly explained to the patient and written consent was taken. Vertigo symptom scale and Vertigo handicap questionnaire were given to the patient and any query regarding understanding the questions were cleared at the same time by the therapist. Therapist explained the method to fill the questionnaire that is to encircle the appropriate option as felt by the patient. Therapist explained the method to fill the questionnaire that is to encircle the appropriate option as felt by the patient. Vertigo symptom scale includes 34 items, which measures 22 symptoms related to vertigo. VSS consists of two sub scales: vertigo severity scale (19 items) and somatic anxiety scale (15 items). To obtain a measure of vertigo severity, simply sum the patient's responses to the following items of VSS: 1 a to $1 e, 4,5,7 a$ to $7 e$, $11,15,18$ a to 18 e. A measure of somatic anxiety can be obtained by summing items $2,3,6,8$ to 10,12 to $14,16,17,19$ to 22 . The scoring in VSS is based upon the frequency during the last year (scored as 0-4 points) i.e.

$0=$ never,

1= A few times (1-3 times a year)

$2=$ several times (4-12 times a year) 
$3=$ quite often (on average, more than once a month)

$4=$ very often (on average, more than once a week)

The total scoring for this scale is 136 points.

The other scale used in this study was vertigo handicap questionnaire (VHQ) it consists of 25 items concern with restriction and disruption of wide range of physical and social activities. Ratings of frequency of disability or handicap are scored (0-4) on a scale of

$0=$ never

$1=$ occasionally

$2=$ sometimes

$3=$ often

4= always.

To obtain the total handicap score (out of 100) simply sum responses of items 1 to 25 of the $\mathrm{VHQ}$, after first reversing the scores on the item no $2,4,6,9,11,14,16,18,21,24$. (So that $0=4$, $4=0$, etc).

The total score range is $0-100$ points and score of 1 and 2 points are less frequent and of 3 and 4 are more frequent. Then the duly filled $q$ uestionnaires were returned to the therapist. The therapist accordingly scored the questionnaires. Out of 41 respondent's one patient's responses was not send for statistical analysis as the questionnaire was half filled. Patients score on VSS and on VHQ was sent for statistical analysis to find out whether increase or decrease in scores of VSS has any correlation with scores on VHQ questionnaire, which will interpret whether the patient with vertigo and its severity are having any impact on their daily living.

Data Analysis: In this study, statistical analysis was done using SPSS (Statistical Package for the Social Sciences) version 20.0.

Data were presented as mean \pm standard deviation (SD) and percentage (P).

Comparison of gender, diagnosis with respect to vertigo handicap questionnaire, total VSS and its dimension scores was done by using $t$ test. Comparison of age groups, gender, and duration with respect to Vertigo handicap questionnaire scores, total VSS and its dimensions scores was done by using one way ANOVA
Correlation between total VSS scores and its dimensions with Vertigo handicap scores by Karl Pearson's correlation coefficient was done. Simple linear regression analysis of Vertigo handicap scores by total VSS scores was done. Multiple linear regression analysis of Vertigo handicap scores by dimensions of total VSS scores was done.

$P$ value $<0.05$ was considered as statistically significant.

\section{RESULTS AND DISCUSSION}

As shown in the table 1 , in our study respondents were of age group ranging from 20-50+ years of age. The mean age of onset of vertigo due to peripheral vestibular disorder was $44.23 \pm 12.15$ years. Previous studies have reported a mean age of $49-52$ years $[9,10,11]$. It has been found that there is an association between advanced age with an odds ratio of 1.8 for every decade. They found that this was not surprising as detachment of otoconia from otolith organs, the prerequisite for BPPV, seems to increase with the age [11].

As shown in the table 2, Total numbers of female in our study were greater than male this was not surprising as previous studies have shown that there is an association between female sex and vestibular vertigo as premenstrual or oral contraceptive- related hormonal change increase the risk for vestibular disorder. Decreased estrogen levels may disturb the internal structure of otoconia or their interconnections and attachments to gelatinous matrix. Otoconia are the deposits of calcium carbonate in the form of composite calcium crystals, and bone contains $99 \%$ of the calcium found in the body. Alternatively, an increase in the concentration of free calcium in the endolymph due to increased calcium resoption may reduce the capacity to dissolve the dislodged otoconia [12]. Idiopathic BPPV is more prevalent in elderly and women. The ratio of female to male is $2-3: 1$ $[11,13]$.

Although there no significant difference in mean age groups among males and females, in females the mean age of onset was more than 45 years, which correlates to study, conductedby jeong et al. The study suggest that (jeong et al) bone mineral density score were decreased 
Table 1: Distribution of samples by age groups and gender.

\begin{tabular}{|c|c|c|c|c|c|c|}
\hline Age groups & Male & $\%$ & Female & $\%$ & Total & $\%$ \\
\hline 20-34yrs & 6 & 66.67 & 3 & 33.33 & 9 & 22.5 \\
\hline 35-49yrs & 5 & 29.41 & 12 & 70.59 & 17 & 42.5 \\
\hline 50+yrs & 6 & 42.86 & 8 & 57.14 & 14 & 35 \\
\hline Total & 17 & 42.5 & 23 & 57.5 & 40 & 100 \\
\hline Mean age & \multicolumn{2}{|c|}{42.76} & \multicolumn{2}{c|}{45.3} & \multicolumn{2}{c|}{44.23} \\
\hline SD age & \multicolumn{2}{|c|}{13.69} & \multicolumn{2}{c|}{11.08} & \multicolumn{2}{c|}{12.15} \\
\hline
\end{tabular}

Table 2: Distribution of samples by diagnosis and gender.

\begin{tabular}{|c|c|c|c|c|c|c|}
\hline Diagnosis & Male & $\%$ & Female & $\%$ & Total & $\%$ \\
\hline BPPV & 16 & 42.11 & 22 & 57.89 & 38 & 95 \\
\hline Meniere's disease & 1 & 50 & 1 & 50 & 2 & 5 \\
\hline Total & 17 & 42.5 & 23 & 57.5 & 40 & 100 \\
\hline
\end{tabular}

Table 3: Distribution of samples by duration and gender.

\begin{tabular}{|c|c|c|c|c|c|c|}
\hline Duration & Male & $\%$ & Female & $\%$ & Total & $\%$ \\
\hline <6 months & 5 & 38.46 & 8 & 61.54 & 13 & 32.5 \\
\hline 6month to 1 year & 7 & 36.84 & 12 & 63.16 & 19 & 47.5 \\
\hline 1-2 year & 2 & 40 & 3 & 60 & 5 & 12.5 \\
\hline$>\mathbf{2}$ year & 3 & 100 & 0 & 0 & 3 & 7.5 \\
\hline Total & 17 & 42.5 & 23 & 57.5 & 40 & 100 \\
\hline
\end{tabular}

Table 4: Comparison of age groups with respect to total VSS and its dimensions scores by one-way ANOVA.

\begin{tabular}{|c|c|c|c|c|c|c|}
\hline \multirow{2}{*}{ Age groups } & \multicolumn{2}{|c|}{ Total VSS } & \multicolumn{2}{|c|}{ Vertigo severity } & \multicolumn{2}{|c|}{ Somatic anxiety } \\
\hline & Mean & SD & Mean & SD & Mean & SD \\
\hline 20-34yrs & 31.44 & 12.69 & 16.56 & 8.73 & 14.89 & 6.99 \\
\hline $35-49 y r s$ & 22.88 & 8.51 & 13 & 6.21 & 9.88 & 4.72 \\
\hline $50+y r s$ & 32.43 & 12.45 & 15.71 & 6.59 & 16 & 8.64 \\
\hline Total & 28.15 & 11.64 & 14.75 & 6.96 & 13.15 & 7.23 \\
\hline F-value & \multicolumn{2}{|c|}{3.4238} & \multicolumn{2}{|c|}{0.9742} & \multicolumn{2}{|c|}{3.4749} \\
\hline p-value & \multicolumn{2}{|c|}{$0.0432 *$} & \multicolumn{2}{|c|}{0.387} & \multicolumn{2}{|c|}{$0.0414^{*}$} \\
\hline \multicolumn{7}{|c|}{ Pair wise comparison of age groups by Tukeys multiple post hoc procedures } \\
\hline $20-34 y r s$ vs $35-49 y r s$ & \multicolumn{2}{|c|}{0.1555} & \multicolumn{2}{|c|}{0.4383} & \multicolumn{2}{|c|}{0.1893} \\
\hline $20-34 y r s$ vs $50+y r s$ & \multicolumn{2}{|c|}{0.9761} & \multicolumn{2}{|c|}{0.957} & \multicolumn{2}{|c|}{0.923} \\
\hline $35-49 y r s$ vs $50+y r s$ & \multicolumn{2}{|c|}{$0.0500 *$} & \multicolumn{2}{|c|}{0.5321} & \multicolumn{2}{|c|}{$0.0450 *$} \\
\hline
\end{tabular}

$* p<0.05$

Table 5: Comparison of age groups with respect to Vertigo handicap scores by one-way ANOVA.

\begin{tabular}{|c|c|c|}
\hline \multirow{2}{*}{ Age groups } & Vertigo handicap scores \\
\cline { 2 - 3 } & Mean & SD \\
\hline 20-34yrs & 48.44 & 16.94 \\
\hline 35-49yrs & 44.47 & 9.97 \\
\hline 50+yrs & 49.43 & 14.63 \\
\hline Total & 47.1 & 13.28 \\
\hline F-value & \multicolumn{2}{|c|}{0.5819} \\
\hline \multicolumn{2}{|c|}{0.5639} \\
\hline p-value & \multicolumn{2}{|c|}{0.7545} \\
\hline 20-34yrs vs 35-49yrs & \multicolumn{2}{|c|}{0.984} \\
\hline 20-34yrs vs 50+yrs & \multicolumn{2}{|c|}{0.5671} \\
\hline 35-49yrs vs 50+yrs & \\
\hline
\end{tabular}

Table 6: Comparison of male and females with respect to total VSS and its dimensions scores by t test.

\begin{tabular}{|c|c|c|c|c|c|}
\hline Variable & Gender & Mean & SD & t-value & p-value \\
\hline \multirow{2}{*}{ Total VSS } & Male & 28.94 & 13.15 & 0.3653 & 0.7169 \\
\cline { 2 - 6 } & Female & 27.57 & 10.66 & & \\
\hline \multirow{2}{*}{ Vertigo severity } & Male & 16.53 & 7.87 & 1.4082 & 0.1672 \\
\cline { 2 - 6 } & Female & 13.43 & 6.04 & & \\
\hline \multirow{2}{*}{ Somatic anxiety } & Male & 12.41 & 7.61 & -0.5501 & 0.5854 \\
\cline { 2 - 6 } & Female & 13.7 & 7.06 & & \\
\hline
\end{tabular}

Table 7: Comparison of male and females with respect to Vertigo handicap scores by $t$ test.

\begin{tabular}{|c|c|c|c|c|}
\hline Gender & Mean & SD & t-value & p-value \\
\hline Male & 47.59 & 14.22 & \multirow{2}{*}{0.1974} & \multirow{2}{*}{0.8445} \\
\hline Female & 46.74 & 12.86 & & \\
\hline
\end{tabular}


Table 8: Comparison of durations with respect to total VSS and its dimensions scores by one-way ANOVA.

\begin{tabular}{|c|c|c|c|c|c|c|}
\hline \multirow{2}{*}{ Duration } & \multicolumn{2}{|c|}{ Total VSS } & \multicolumn{2}{c|}{ Vertigo severity } & \multicolumn{2}{c|}{ Somatic anxiety } \\
\cline { 2 - 7 } & Mean & SD & Mean & SD & Mean & SD \\
\hline <6 months & 25.85 & 10.21 & 13.38 & 5.74 & 12.46 & 7.42 \\
\hline 6month to 1 year & 26.21 & 12.19 & 13.42 & 7.76 & 12.79 & 6.65 \\
\hline 1-2 year & 33.8 & 10.18 & 20 & 3.08 & 11.8 & 7.92 \\
\hline$>2$ year & 41 & 8.72 & 20.33 & 6.35 & 20.67 & 8.5 \\
\hline Total & 28.15 & 11.64 & 14.75 & 6.96 & 13.15 & 7.23 \\
\hline F-value & \multicolumn{2}{|c|}{2.1251} & \multicolumn{2}{c|}{2.1706} & \multicolumn{2}{c|}{1.2133} \\
\hline p-value & \multicolumn{2}{|c|}{0.1141} & \multicolumn{2}{c}{0.1084} & \multicolumn{2}{c}{0.3189} \\
\hline
\end{tabular}

Table 9: Comparison of durations with respect to Vertigo handicap scores by one-way ANOVA.

\begin{tabular}{|c|c|c|}
\hline \multirow{2}{*}{ Duration } & \multicolumn{2}{|c|}{ Vertigo handicap scores } \\
\cline { 2 - 3 } & Mean & SD \\
\hline <6 months & 45.46 & 12.91 \\
\hline 6month to 1 year & 43.95 & 12.95 \\
\hline 1-2 year & 54.4 & 10.29 \\
\hline$>$ 2 year & 62 & 11.79 \\
\hline Total & 47.1 & 13.28 \\
\hline F-value & \multicolumn{2}{|c|}{2.4251} \\
\hline p-value & \multicolumn{2}{|c|}{0.0815} \\
\hline
\end{tabular}

Table 10: Correlation between total VSS scores and its dimensions with Vertigo handicap scores by Karl Pearson's correlation coefficient method.

\begin{tabular}{|c|c|c|c|}
\hline \multirow{2}{*}{ Variables } & \multicolumn{3}{|c|}{ Correlation between Vertigo } \\
\cline { 2 - 4 } & r-value & t-value & p-value \\
\hline Total VSS & 0.5452 & 4.0087 & $0.0003^{*}$ \\
\hline Vertigo severity & 0.4532 & 3.1344 & $0.0033^{*}$ \\
\hline Somatic anxiety & 0.442 & 3.0379 & $0.0043^{*}$ \\
\hline
\end{tabular}

$* p<0.05$

Table 11: Simple linear regression analysis of Vertigo handicap scores by total VSS scores.

\begin{tabular}{|c|c|c|c|c|}
\hline $\begin{array}{c}\text { Independent } \\
\text { variables }\end{array}$ & Estimates & $\begin{array}{c}\text { SE of } \\
\text { estimate }\end{array}$ & t-value & p-level \\
\hline Intercept & 42.4231 & 2.9892 & 14.1921 & $0.00001^{*}$ \\
\hline Total VSS & 4.9231 & 2.3271 & 2.1155 & $0.0410^{*}$ \\
\hline
\end{tabular}

Table 12: Multiple linear regression analysis of Vertigo handicap scores by dimensions of total VSS scores.

\begin{tabular}{|c|c|c|c|c|}
\hline Independent variables & Estimates & SE of estimate & $t$-value & $p$-level \\
\hline Intercept & 29.4537 & 4.8134 & 6.119 & $0.00001^{*}$ \\
\hline Vertigo severity & 0.5726 & 0.2588 & 2.2128 & $0.0332^{*}$ \\
\hline Somatic anxiety & 0.1036 & 0.4331 & 0.2392 & 0.8123 \\
\hline$R=0.5461, R^{2}=0.2982$, Adjusted $R^{2}=0.2603, F(2,37)=7.8640$ & $p<0.05, S$, Std.Error \\
of estimate: 11.421
\end{tabular}

${ }^{*} p<0.05$

in women $>45$ years suggesting the involvement of deranged calcium metabolism in idiopathic BPPV and a significant association between osteopenia/ osteoporosis and idiopathic BPPV [14].

As shown in table no 4 , out of 40 respondents $38(95 \%)$ individuals were diagnosed to have BPPV and $2(5 \%)$ were of Meniere's disease. BPPV is the most common vestibular disorder followed by Meniere's disease $[9,11,15,16]$. Only two participants were found to have Meniere disease, but this was not surprising because Meniere disease is a rare condition. The numbers of patients with MD were less in our study as diagnosis of MD requires a wide differential diagnosis and is not easy to detect [17].

As shown in table no 5, duration majority of the patients in our study fall into group $A$ and $B$, this can be attributed to BPPV being a self-limiting condition, it resolves spontaneously within 6 to 12 months.

As shown in table no 6, When the age groups were compared with respect to the total VSS and its dimension scores i.e. vertigo severity scale and somatic anxiety scale by one way Annova, $p$ value was found to be significant in total VSS scores and somatic anxiety scores for the age group of 35-49 years vs $50+$ years. This indicated that the somatic anxiety contributed more towards increase in the total VSS. The increase in somatic anxiety scores could be because the numbers of female patients were more in the study between $35-49$ years (12) and 50+ years (8). As the female patients, have higher psychological impact leading to elevated anxiety levels [18].

When the age group was compared with respect to $\mathrm{VHQ}$, no significant difference was found.

Previous studies have also shown that women have higher psychological distress than men do as they suffer with greater psychological impact 
from vertigo than men as well as the prevalence of such disease is higher in women but still comparison of gender with VSS and its dimension scores and VHQ did not show any statistically significant difference $[18,19]$.

When VSS and its dimension scores, \& VHQ were compared with duration, no significant difference was found. Generally, it has been found that the duration of the disease is an important factor for quality of life. BPPV usually resolves within 6 to 12 months spontaneously as it is selflimiting disorder, but spontaneous remissions are common and recurrences may trouble the patient intermittently or for years. The longer the patients' lives with the chronicity of the disease or the illness more frequently they were found to be reporting somatic anxiety, decrease psycho-social functioning, and disease specific handicap. Thus, it may indicate that the patient with peripheral vestibular disorder may have difficulty in coping with disease specific symptoms. In Mores and Johnson's model for acute and chronic illness, four stages have been identified. The stages are stage of uncertainty and suspicion, the stage of disruption in which the decision to seek help creates or may include higher level of stress and dependence, the stage of striving for recovery and control, often during treatment, and finally the stage of acceptance of the illness and its consequences and a restoration of wellbeing. According to this model and in a view that majority of the patients were in the diagnostic phase so they were in the second stage of the illness [20].

In present study, correlation was found between VSS and its dimension scores with respect to VHQ. Total VSS scale correlated with the handicap scores in the regression analysis with $\mathrm{VHQ}$ as a dependent variable. Similarly, sub scale of vertigo severity also correlated with the VHQ in multiple regression analysis. Thus, severity of symptoms of vertigo was the foremost predictor of the level of handicap. Change in the severity of vertigo could affect handicap/QOL because of physical and psychological factors. The physical factors contributing to severity of vertigo are feeling that things are spinning or moving around, unsteadiness so severe that you actually fall, nausea, feeling of being lightheaded/swimmy or giddy, vomiting, unable to stand or walk properly without support, feeling unsteady about to lose balance. In the present study, $63 \%$ of the participants reported difficulty in standing and walking without support and $80 \%$ were feeling unsteady. Several studies show that vestibular disorders result in reduced postural control with instability during ambulation and during transitional activities such as moving, from lying to sitting and sitting to standing. As seen in BPPV vertigo is induced by the change in head position with respect to gravity example getting out of the bed, rolling over in the bed, tilting their head back, or bending forward. Symptoms of BPPV are found to be varying among patients, and may manifest with non-specific dizziness, postural instability, lightheadedness and nausea [15]. Vertigo, unsteadiness and imbalance experienced by patients are linked with an increased incidence of fall. The evidence is therefore clear that vertigo and related vestibular troubles can dramatically affect patients' QOL and even totally incapacitate the individual [21].

Vertigo is a psychologically disabling symptom due to three reasons: as people are unable to locate its source, next arousal of vertigo is unforeseeable so generally, patients have a fear of the next episode of vertigo so it is accompanied by anxiety and panic attacks, and lastly it induces a profound involvement of body and mental sensations. ${ }^{18}$ In present study 32 (80\%) felt that there is something seriously wrong with them. This fact can be explained by the reason that vertigo is a psychologically disabling condition as the patients are unable to locate their source of illness. Complaints of vertigo are often accompanied by the elevated level of the anxiety and emotional disturbances. In the present study out of 40 participants, 36 (90\%) were getting anxious during an attack of vertigo and 32 (80\%) were depressed due to vertigo. In a study by Yardley, depression was independently related to handicap, they found that the recent mood change i.e. in the form of negative mood may at first result from vertigo later it act as a factor maintaining or exacerbating level of handicap. Depressed sense of energy and enthusiasm can lead to the restriction in activity [4].

In the present study majority $30(75 \%)$ patients 
were finding difficulty in their daily activities like e.g. shopping, gardening, jobs around the house due to vertigo. Vertigo has an impact on life of the patient during asymptomatic period due to the anticipation of unpredictable episode of vertigo itself 27 (67.5\%) patients were avoiding there plans as they were afraid of spoiling things of others. $32(80 \%)$ patients were finding the attacks as frightening. Thus, handicap scores can be taken to represent the psychosocial impact of vertigo which can give rise to these complains. A number of the psychosomatic and somatopsychic mechanisms might be responsible for this relationship. Psychosomatic processes include increased awareness and reporting of sensations of disorientation in anxious patients who experience minor episodes of vertigo. Somatopsychic explanations include the intrinsically frightening nature of vertigo attacks and their disruption of daily activities. Thus, people with a previously normal psychiatric history frequently develop signs of anxiety, panic and agoraphobia after the onset of vestibular disorder [4,7].

Thus psychological factor play an important role in maintaining or increasing the handicap or perception of vertigo. If handicap is found to be prevalent then it is important to explain to the patient during the counseling phase in order to reduce the incidence rate of anxiety. If the patients are not able to control his /her emotions, the psychological assistance is helpful and it should be started as soon as possible. Sometimes altered synchrony is found between vestibular test results and patient complains thus inevitably suggests the suspicion towards the psychological element in the disorder. Thus, vertigo severity can be used as a predictor to find the level of handicap in the patients with vertigo due to peripheral vestibular disorder.

\section{CONCLUSION}

We aimed in this study to correlate between vertigo symptom scale and vertigo handicap questionnaire in patients with vertigo due to peripheral vestibular disorder. There were total 40 patients included in the study out of which $42.50 \%$ were male and $57.50 \%$ were female out of which $95 \%$ cases were of BPPV and $5 \%$ cases of Meniere's disease. After getting the filled VSS and VHQ from the patients, the data was analyzed and the results were taken which showed there is correlation between VSS and VHQ in patients with peripheral vestibular disorder. Relationship was found between symptoms, anxiety and handicap because of the proximal somatopsychic and psychosomatic mechanisms.

\section{ACKNOWLEDGEMENTS}

First of all, I thank God, by the grace of whom I have all that I need. I express my deep sense of gratitude to Dr. Ravi Savadatti Principal S.D.M. College of Physiotherapy for his support all through the study period. I express my gratitude towards my research guide Dr. Sharmila Dudhani, Assistant Professor \& PG Guide, S.D.M. College of Physiotherapy, Dharwad, who has given me her valuable guidance without which perhaps I would not have completed my research work. My special gratitude and thanks to my lovely Grandmother, My parents, My sisters. I would also like to thank all the Neurophysicians and ENT department of SDMCMSH without whom, I would not have been able to complete the task. Last but not the least to all the patients who participated in the study, without whom this task would not have been possible.

\section{Conflicts of interest: None}

\section{REFERENCES}

[1]. Rogers C, Wet J, Gina A, Louw L, Makhoba M, Tacon L. The translation of the Vertigo Symptom Scale into Afrikaans: A pilot study. SAJCD 2011;58:6-12.

[2]. Wilhelmsen K, Strand L, NordahI S, Eide G, Ljunggren A. Psychometric properties of the Vertigo symptom scale - Shortform. BMC Ear, Nose and Throat Disorders 2008;8:1-9.

[3]. Yardley L, Medina SM, Jurado CS et al. Relationship between physical and psychosocial dysfunction in Mexican patients with vertigo: a cross-cultural validation of the vertigo symptom scale. J Psychosom Res 1999;46:63-74.

[4]. Yardley L, Masson E, Verschuur C et al. Symptoms, anxiety and handicap in dizzy patients: development of the vertigo symptom scale. J Psychosomatic Research 1992;36:731-41.

[5]. Zainun Z, Zakaria N, Sidek D. Clinical use of Malay Version of Vertigo Symptom Scale (MVVSS) in Patients with Peripheral Vestibular Disorder. Med J Malaysia 2012;67(4):386-389.

[6]. Duracinsky M, Mosnier I, Bouccara D. Literature Review of Questionnaires Assessing Vertigo and Dizziness, and Their Impact on Patients' Quality of Life. International Society for Pharmacoeconomics and Outcomes Research 2007;10(4):273-284. 
[7]. Yardley L, Putamen J. Quantitative analysis of factors contributing to handicap and distress in vertiginous patients: a questionnaire study. Clinical Otolaryngology \& Allied Sciences 1992;17(3):231236.

[8]. Neuhauser $H$, Radtke A, Brevern M, Lezius F, Feldmann $M$, Lempert T. Burden of Dizziness and Vertigo in the Community. Arch intern medicine 2008;168(19):2118-2124.

[9]. Salvinelli F, Firrisi L. Benign paroxysmal positional vertigo: diagnosis and treatment. La Clinica Terapeutica 2004;155(9):395-400.

[10].Mizukoshi K, Watanabe Y, Shojaku H, Okubo J, Watanabe I. Epidemiological studies on benign paroxysmal positional vertigo in Japan. Acta Otolaryngology Suppl 1988;447:67-72.

[11]. Brevern M, Radtke A, Lezius F, Feldmann M, Ziese T, Lempert T, Neuhauser H. Epidemiology of benign paroxysmal positional vertigo: a population based study. J Neurol Neurosurgery Psychiatry 2007;78:710-715.

[12]. Vibert D, Kompis M, Hausler R. Benign paroxysmal positional vertigo in older women may be related to osteoporosis and osteopenia. Ann Otol Rhinol Laryngol 2003;112:885-889.

[13]. Baloh RW, Honrubia V, Jacobson K. benign positional vertigo: clinical and oculographic features in 240 cases. Neurology 1987;37(3)371-378.

[14]. Jeong SH, Choi SH, Kim JY, Koo JW, Kim HJ, Kim JS. Osteopenia and osteoporosis in idiopathic benign positional vertigo. Neurology 2009;72:1069-1076.
[15]. Lee S, Kim J. Benign paroxysmal Positional Vertigo. J ClinNeurology 2010;6:51-63.

[16]. Sekine K, Sato G, Takeda N. Incidence of vertigo and dizziness disorders at a university hospital. Nihon Jibiinkoka GakkaiKaiho 2005;108(9):842-849.

[17]. Brevern M, Radtke A, Lezius F, Feldmann M, Ziese T, Lempert T, Neuhauser $H$. Screening for Meniere's disease in the general population: the needle in the haystack. Acta Otolaryngol 2008;128(3):272-276.

[18]. Monzani D, Casolari L, Guidetti G, Rigatelli M. Psychological distress and disability in patients with vertigo. J Psychosomatic Research 2001;50(6):319323.

[19]. Yardley L. Contribution of symptoms and beliefs to handicap in people with vertigo: A longitudinal study. Br J Clin Psychology 1994;33(1):101-113.

[20]. Mendel B, Bergenius J, Langius A. Dizziness symptom severity and impact on daily living as perceived by patients suffering from peripheral vestibular disorder. Clinical Otolaryngology Allied science 1999;24(4):286-93.

[21]. Mira E. Improving the quality of life in patients with vestibular disorders: the role of medical treatments and physical rehabilitation. International journal of clinical practice 2008;62(1):109-114.

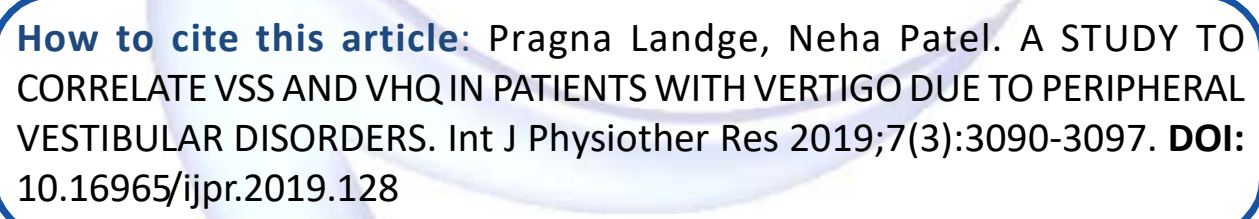

How to cite this article: Pragna Landge, Neha Patel. A STUDY TO CORRELATE VSS AND VHQ IN PATIENTS WITH VERTIGO DUE TO PERIPHERAL VESTIBULAR DISORDERS. Int J Physiother Res 2019;7(3):3090-3097. DOI: 10.16965/ijpr.2019.128 